\title{
MEAN-VARIANCE HEDGING FOR STOCHASTIC VOLATILITY MODELS
}

\author{
FRANCESCA BIAGINI, PAOLO GUASONI, AND MAURIZIO PRATELLI
}

\begin{abstract}
In this paper we discuss the tractability of stochastic volatility models for pricing and hedging options with the mean-variance hedging approach. We characterize the variance-optimal measure as the solution of an equation between Doleans exponentials: explicit examples include both models where volatility solves a diffusion equation, and models where it follows a jump process.

We further discuss the closedness of the space of strategies.
\end{abstract}

\section{INTRODUCTION}

The mean-variance hedging approach to pricing and hedging contingent claims was introduced (in the martingale case) by Föllmer/Sondermann (1986) : subsequent extensions to the general semimartingale case were done by Duffie/Richardson (1991) , Schweizer (1992, 1996) , Schäl (1994), Gouriéroux et al. (1998), Pham et al. (1997) and Rheinländer/Schweizer (1997). The paper of Schweizer (1999) contains a general overview of the subject, and a complete bibliography.

The aim of this paper is to analyse the mean-variance hedging criterion in stochastic volatility models : we develop a general framework (introduced by Föllmer/Schweizer (1991) ) where a stochastic volatility model is seen as a model with incomplete information.

This model would be complete with respect to some larger filtration (usually including all information on past and future volatility), but not under the filtration available to the hedging agent (who usually observes only the asset price history). This framework is general enough to include both the diffusion models (such as HullWhite, Heston, Stein and Stein, only to mention a few), and less common models where volatility jumps.

We begin our analysis with a characterization of the set of equivalent martingale measures in terms of Doléans exponentials: this provides a one-one correspondence between equivalent martingale measures and a class of predictable processes. Exploiting results of Schweizer (1996) and Delbaen/Schachermayer (1996), we then identify the variance-optimal martingale measure as the solution of an equation involving exponential martingales.

Our results are illustrated by several examples: the detailed analysis of all these examples can be found in Biagini/Guasoni (1999).

In the case of diffusion stochastic volatility models we recover some results of Laurent/Pham (1999) with a different method: in fact, while they use dynamic programming techniques, we essentially focus on stochastic integration. Also the

1991 Mathematics Subject Classification. 60H30, 90A09; JEL Classification: G10.

Key words and phrases. Hedging in incomplete markets, stochastic volatility models, meanvariance optimal measure, change of numéraire. 
recent paper by Heath et al. (1999) contains a detailed analysis of the mean-variance hedging criterion (compared to the locally risk-minimizing criterion) in stochastic volatility models.

In order to keep notations simple, we only consider one-dimensional models; however our results can easily be extended to the multidimensional case.

\section{Statement of the PRoblem}

For all definitions on stochastic integration and martingale representation, we refer to Protter (1990) and Dellacherie/Meyer (1982) (in particular, all filtrations are supposed to satisfy the so-called usual hypothesis ).

We have two complete filtered probability spaces denoted by $\left(\Omega, \mathcal{F}^{W}, \mathcal{F}_{t}^{W}, P^{W}\right)$ and $\left(E, \mathcal{E}, \mathcal{E}_{t}, P^{E}\right)$. We assume that $W_{t}$ is a standard Brownian Motion on $\Omega=$ $C([0, T], \mathbb{R}), P^{W}$ is the standard Wiener measure, $\mathcal{F}^{W}=\mathcal{F}_{T}^{W}$, and $\mathcal{F}_{t}^{W}$ is the $P^{W}$ augmentation of the filtration generated by $W$.

We have two assets: the risky asset $S_{t}$, and the riskless asset $B_{t}=\exp \left(\int_{0}^{t} r_{s} d s\right)$, where $r_{t}$ is a deterministic function. The risky asset is represented by a process $S_{t}(w, \eta)$ on the product space $\Omega \times E$, whose dynamics is given by the following equation:

$$
\left\{\begin{array}{l}
d S(t, \omega, \eta)=S(t, \omega, \eta)\left(\mu(t, \omega, \eta) d t+\sigma(t, \omega, \eta) d W_{t}(\omega)\right) \\
S(0)=S_{0}
\end{array}\right.
$$

We shall make the following assumptions:

i) on the space $E$ we have a (possibly $d$-dimensional) martingale $M$ which has the predicable representation property with respect to the filtration $\left(\mathcal{E}_{t}\right)_{t \in[0, T]}$.

ii) the information available at time $\mathrm{t}$ is given by the filtration $\mathcal{F}_{t}^{W} \otimes \mathcal{E}_{t}$.

iii) the probability $P$ on $\Omega \otimes E$ is the product probability $P^{W} \otimes P^{E}$.

Remark 1.1. In many applications, the most natural filtration available to the agent is the one generated by $S$ : let us see how $i i$ ) translates in this case. If $\sigma$ has a rightcontinuous version, it is $\mathcal{F}_{t}^{S}$-adapted: in fact we recall that (see Föllmer/Schweizer (1991) page 410)

$$
\langle S\rangle_{t}=\int_{0}^{t} \sigma_{s}^{2} S_{s}^{2} d s=\lim _{\sup _{i}\left|t_{i+1}-t_{i}\right| \rightarrow 0} \sum\left(S_{t_{i+1}}-S_{t_{i}}\right)^{2}
$$

is $\mathcal{F}_{t}^{S}$-adapted. If $\mu(t, \omega, \eta)$ is also $\mathcal{F}_{t}^{S}$-adapted, it is easy to see that the filtration generated by $S$ coincides with the one generated by $(W, \mu, \sigma)$. Therefore the assumption ii) boils down to

$$
\mathcal{F}_{t}^{W, \mu, \sigma}=\mathcal{F}_{t}^{W} \otimes \mathcal{E}_{t}
$$

Remark 1.2. Since the technicalities involved in the definition above may hide the idea of incomplete information, we provide a simple explanation. This market would be complete if the agent had access to the (larger) filtration $\widetilde{\mathcal{F}}_{t}=\mathcal{F}_{t} \otimes \mathcal{E}$, which contains at any time all the information on past and future drift and volatility. As pointed out by Föllmer/Schweizer (1991), this is a consequence of the fact that all 
$\widetilde{\mathcal{F}}_{t}$-martingales can be written in the form

$$
N_{t}(\omega, \eta)=N_{0}(\eta)+\int_{0}^{t} H_{s}(\omega, \eta) d W_{s}(\omega)
$$

for some $\widetilde{\mathcal{F}}$-predictable process $H$. This result is an exercise on stochastic integration: we provide a proof in the appendix (Proposition 5.1), for the sake of completeness.

The discounted value of the risky asset follows the equation:

$$
\left\{\begin{array}{l}
d X_{t}=X_{t}\left(\left(\mu_{t}-r_{t}\right) d t+\sigma_{t} d W_{t}\right) \\
X_{0}=S_{0}
\end{array}\right.
$$

We assume $\mu$ and $\sigma$ are such that $X_{t} \in L^{2}(P)$ for all $t \in[0, T]$, and denote by $\lambda_{t}(\omega, \eta)=\frac{\mu(t, \omega, \eta)-r(t)}{\sigma(t, \omega, \eta)}$ the so-called market price of risk.

Example 1.3. This example was introduced by Harrison/Pliska (1981), and investigated later by Föllmer/Schweizer ( (1991), page 142).

$\mu_{t}$ and $\sigma_{t}$ are constant until a fixed time $t_{0}$, then they jump simultaneously, the pair $(\mu, \sigma)$ having two possible outcomes. In other words

$$
\left\{\begin{array}{l}
\mu_{t}(\eta)=1_{\left\{t<t_{0}\right\}} \mu+1_{\left\{t \geq t_{0}\right\}} \mu_{\eta} \\
\sigma_{t}(\eta)=1_{\left\{t<t_{0}\right\}} \sigma+1_{\left\{t \geq t_{0}\right\}} \sigma_{\eta}
\end{array}\right.
$$

where $E=\{0,1\}, \mathcal{E}_{t}=\{\emptyset, E\}$ for $t<t_{0}$ and $\mathcal{E}_{t}=\mathcal{P}(E)$ for $t \geq t_{0}$. A fundamental martingale is given by $M_{t}=1_{\left\{t \geq t_{0}\right\}}\left(1_{\{\eta=1\}}-p\right)$, where $p=P(\eta=1)$.

This example was generalized by Föllmer/Leukert (1999), where the values of $\mu_{t}$ and $\sigma_{t}$ after the jump time $t_{0}$ have a continuous distribution: in this case $E=\mathbb{R}$ and the martingale $M$ has to be replaced by a random measure ( see Biagini/Guasoni (1999) for details).

Example 1.4. The previous example can be extended in several ways: we consider in particular a model proposed in discrete time fashion in RiskMetrics Monitor (see Zangari (1996) ) as an improvement of the standard lognormal model for calculating Value at Risk. More precisely, we have multiple independet jumps at fixed equispaced time intervals. We can set $E=\{0,1\}^{n}$ and, denoting $\eta=\left\{a_{1}, \ldots, a_{n}\right\}, \mathcal{E}_{t}$ is equal to the parts of $\left\{a_{i}\right\}_{t_{i} \leq t}$ ( where $t_{i}=i \frac{T}{n+1}$ ). One obtains the following dynamics:

$$
\left\{\begin{array}{l}
\mu_{t}(\eta)=1_{\left\{t<t_{1}\right\}} \mu+\sum_{i} 1_{\left\{t_{i} \leq t<t_{i+1}\right\}} \mu_{a_{i}}+1_{\left\{t \geq t_{n}\right\}} \mu_{a_{n}} \\
\sigma_{t}(\eta)=1_{\left\{t<t_{1}\right\}} \sigma+\sum_{i} 1_{\left\{t_{i} \leq t<t_{i+1}\right\}} \sigma_{a_{i}}+1_{\left\{t \geq t_{n}\right\}} \sigma_{a_{n}}
\end{array}\right.
$$

Since in this model $\eta$ is binomially distributed ( in fact the numbers $a_{i}$ are independent and $\left.P\left(a_{i}=1\right)=p\right)$, it is evident the existence of a martingale with the representation property.

Example 1.5. This example was studied in detail by Biagini/Guasoni (1999). We have

$$
\begin{aligned}
\mu_{t}(\eta) & =1_{\{t<\tau\}} \mu_{1}+1_{\{t \geq \tau\}} \mu_{2} \\
\sigma_{t}(\eta) & =1_{\{t<\tau\}} \sigma_{1}+1_{\{t \geq \tau\}} \sigma_{2}
\end{aligned}
$$


where $\tau$ is a stopping time whose law restricted to $[0, T)$ has a density $f$ ( and $\left.P(\tau=T)=1-\int_{0}^{T} f(s) d s\right)$.

In this case, $E=[0, T], \mathcal{E}_{t}=\mathcal{B}([0, t]) \cup(t, T]$, and a fundamental martingale can be found in the form $M_{t}=1_{\{t \geq \tau\}}-a(t \wedge \tau)$, where $a($.$) is an increasing function$ which can be explicitely determined in terms of $f$.

Example 1.6. The previous example can be generalized in the following way: after the jump time $\tau, \mu$ and $\sigma$ have a general probability distribution independent from $\tau$. The space $E$ is, in this case, $[0, T] \times \mathbb{R}$ and the martingale $M$ is replaced by the random measure $\left(\nu-\nu^{p}\right)$, where $\nu^{p}$ is the compensator of the random measure $\nu(\eta, d t, d x)=\epsilon_{(\tau(\eta), \alpha(\eta))}(d t, d x)$ and $\alpha(\eta)=\lambda^{2}(\eta)-\lambda_{1}^{2}$.

Example 1.7. A number of diffusion stochastic volatility models have been proposed in the literature, most of them being particular cases of the following

$$
\left\{\begin{array}{l}
d X_{t}=\sigma\left(t, X_{t}, v_{t}\right) X_{t}\left(\lambda\left(t, X_{t}, v_{t}\right) d t+d W_{t}^{1}\right) \\
d v_{t}=\alpha\left(t, X_{t}, v_{t}\right) d t+\beta\left(t, X_{t}, v_{t}\right) d W_{t}^{1}+\gamma\left(t, X_{t}, v_{t}\right) d W_{t}^{2}
\end{array}\right.
$$

where $W^{1}$ and $W^{2}$ are two independent Brownian Motions.

We set $E=C([0,1], \mathbb{R})$, and $\mathcal{E}_{t}$ is the augmentation of the filtration generated by $W_{t}^{2}$ : the natural choice for a martingale with the representation property on $E$ is clearly $W^{2}$.

In the general framework described above, an agent wishes to hedge a certain European option $H$ expiring at a fixed time $T$ : his goal is to minimize the risk, defined as the variance of the tracking error at expiration. Therefore we look for a solution to the minimum problem

$$
\min _{\substack{c \in \mathbb{R} \\ \theta \in \Theta}} E\left[\left(H-c-G_{T}(\theta)\right)^{2}\right]
$$

where

$$
G_{t}(\theta)=\int_{0}^{t} \theta_{s} d X_{s} \quad \text { and } \quad \Theta=\left\{\theta \in L(X), G_{t}(\theta) \in \mathcal{S}^{2}(P)\right\}
$$

Here $L(X)$ denotes the space of $X$-integrable $\mathcal{F}_{t}$-predictable processes, and $\mathcal{S}^{2}$ the space of semimartingales $Y$ decomposable as $Y=Y_{0}+M+A$, where $M$ is a squareintegrable martingale, and $A$ is a process of square-integrable variation.

Definition 1.8. We define the following spaces of signed martingale measures

$$
\begin{aligned}
& \mathcal{M}_{s}=\left\{Q \ll P: X_{t} \text { is a } Q \text {-local martingale }\right\} \\
& \mathcal{M}_{e}=\left\{Q \in \mathcal{M}_{s}, Q \sim P\right\} \\
& \mathcal{M}_{s}^{2}=\left\{Q \in \mathcal{M}_{s}, \frac{d Q}{d P} \in L^{2}(P)\right\} \\
& \mathcal{M}_{e}^{2}=\mathcal{M}_{e} \cap \mathcal{M}_{s}^{2}
\end{aligned}
$$

If $Q$ is a signed probability with density $Z$ with respect to $P$, by definition $X_{t}$ is a $Q$-martingale if $X_{t} Z_{t}$ is a $P$-martingale, where $Z_{t}=E[Z \mid \mathcal{F}]$.

The existence of a minimizer for (1) was shown for any $H \in L^{2}(P)$ independently by 
Gouriéroux et al (1998) and Rheinländer/Schweizer (1997) under the two standing assumptions (which need to be checked for each particular model):

i) $\mathcal{M}_{e}^{2} \neq \emptyset$;

ii) $G_{T}(\Theta)$ is closed.

While $i$ ) is equivalent to a no-arbitrage condition (see Delbaen/Schachermayer (1996)) and holds for very general models, $i i$ ) often fails even for models commonly used in practice. However we shall return to this issue later.

If (1) has a solution, the optimal value for $c$ can be written as

$$
c=\widetilde{E}[H]
$$

where $\widetilde{E}$ denotes the expectation under a new signed measure $\widetilde{P}$, the so-called variance-optimal martingale measure. By definition, $\widetilde{P}$ is the element of minimal norm in $\mathcal{M}_{s}^{2}$ (which evidently exists as soon as $\mathcal{M}_{s}^{2} \neq \emptyset$ ): see for instance Schweizer (1996) for further details.

Our first step towards an explicit formula for $\frac{d \widetilde{P}}{d P}$ is the characterization of the set $\mathcal{M}_{e}^{2}$ of the square-integrable equivalent martingale measures. We start by recalling the following:

Definition 1.9. The Doléans exponential $\mathcal{E}(Z)$ of a semimartingale $Z$ is defined as

$$
\mathcal{E}(Z)_{t}=\exp \left(Z_{t}-\frac{1}{2}\left\langle Z^{c}\right\rangle_{t}\right) \prod_{s \leq t}\left(1+\Delta Z_{s}\right) \exp \left(-\Delta Z_{s}\right)
$$

where $Z^{c}$ denotes the continous part of $Z$, while $\Delta Z_{s}=Z_{s}-Z_{s^{-}}$.

We prove now the following lemma.

Lemma 1.10. Let $Z_{t}$ be a local martingale with $Z_{0}=1$. The following conditions are equivalent:

i) $Z_{t} X_{t}$ is a local martingale

ii) $Z_{t}=\mathcal{E}\left(-\int_{0}^{\cdot} \lambda_{s} d X_{s}\right)_{t}\left(1+\int_{0}^{t} k_{s} d M_{s}\right)$ for some predictable process $k_{s}$ such that the stochastic integral $\int_{0}^{t} k_{s} d M_{s}$ is a local martingale.

Proof. We recall that the pair $(W, M)$ has the predictable representation property (see Proposition 5.2). Therefore

$$
Z_{t}=1+\int_{0}^{t} h_{s} d W_{s}+\int_{0}^{t} k_{s} d M_{s}
$$

By Itô's formula, we have

$$
d\left(Z_{t} X_{t}\right)=\left[Z_{t-}\left(\mu_{t}-r_{t}\right)+h_{t} \sigma_{t}\right] X_{t} d t+\left[Z_{t-} \sigma_{t} X_{t}+h_{t} X_{t}\right] d W_{t}+k_{t} X_{t} d M_{t}
$$

The process $\left(Z_{t} X_{t}\right)$ is a local martingale if and only if $h_{t}=-\frac{\mu_{t}-r_{t}}{\sigma_{t}} Z_{t-}$. More precisely, if $\lambda_{t}=\frac{\mu_{t}-r_{t}}{\sigma_{t}}, Z_{t}$ satisfies the following stochastic differential equation:

$$
d Z_{t}=-\lambda_{t} Z_{t-} d W_{t}+k_{t} d M_{t}
$$


which has a unique solution (see Protter (1990) for details). It can easily be verified that $Z_{t}=\mathcal{E}\left(-\int_{0}^{\cdot} \lambda_{s} d X_{s}\right)_{t}\left(1+\int_{0}^{t} k_{s} d M_{s}\right)$ is the solution of the above equation.

If $Z_{T}$ is strictly positive, then $N_{t}=1+\int_{0}^{t} k_{s} d M_{s}$ can be written as the Doléans exponential $N_{t}=\mathcal{E}\left(-\int_{0}^{\cdot} \frac{k_{s}}{N_{s-}} d M_{s}\right)_{t}$.

An immediate consequence of the above lemma is the characterization of $\mathcal{M}_{s}^{2}$ and $\mathcal{M}_{e}^{2}$.

Proposition 1.11. 1. For every $Q \in \mathcal{M}_{s}^{2}$

$$
\frac{d Q}{d P}=\mathcal{E}\left(-\int_{0}^{\cdot} \lambda_{t}(\omega, \eta) d W_{t}\right)_{T}\left(c+\int_{0}^{T} k_{t} d M_{t}\right)
$$

where $k_{t}$ is a process such that the above expression is square integrable.

2. For every $Q \in \mathcal{M}_{e}^{2}$

$$
\frac{d Q}{d P}=\mathcal{E}\left(-\int_{0}^{\cdot} \lambda_{t}(\omega, \eta) d W_{t}\right)_{T} \mathcal{E}\left(\int_{0}^{\cdot} k_{t}(\omega, \eta) d M_{t}\right)_{T}
$$

with $k_{t}$ such that $k_{t} \cdot \Delta M_{t}>-1$ and $\mathcal{E}\left(-\int_{0}^{\cdot} \lambda_{s} d W_{s}+k_{s} d M_{s}\right)_{t}$ is a squareintegrable martingale.

Recall that $\mathcal{E}\left(-\int_{0}^{\cdot} \lambda_{s} d W_{s}+k_{s} d M_{s}\right)_{t}=\mathcal{E}\left(-\int_{0}^{\cdot} \lambda_{s} d W_{s}\right)_{t} \mathcal{E}\left(\int_{0}^{\cdot} k_{s} d M_{s}\right)_{t}$ since $[W, M]=$ 0 (see Protter (1990) pag. 79). Condition $k_{t} \cdot \Delta M_{t}>-1$ guarantees the positivity of $\mathcal{E}\left(\int_{0}^{\cdot} k_{t} d M_{t}\right)_{T}$.

Remark 1.12. A similar characterization holds for the probabilities $Q \ll P$ such that $X_{t}$ is a $Q$-martingale with respect to the enlarged filtration $\tilde{\mathcal{F}}_{t}$ : more precisely

$$
\frac{d Q}{d P}=G(\eta) \mathcal{E}\left(-\int_{0}^{\cdot} \lambda_{s}(\eta) d W_{s}\right)_{T}
$$

with $G$ such that the above expression is square integrable and $E[G]=1$. $Q$ is a true probability if $G>0$.

Before we find an equation to identify $\widetilde{P}$, we need another

Definition 1.13. We define the two processes $\widehat{W}_{t}$ and $W_{t}^{*}$ as follows:

$$
\begin{gathered}
\widehat{W}_{t}=W_{t}+\int_{0}^{t} \lambda_{s} d s \\
W_{t}^{*}=W_{t}+2 \int_{0}^{t} \lambda_{s} d s
\end{gathered}
$$

Remark 1.14. By the theorem of Girsanov, if $\mathcal{E}\left(-\int_{0}^{\cdot} \lambda_{s} d W_{s}\right)_{t}$ and $\mathcal{E}\left(-2 \int_{0}^{\cdot} \lambda_{s} d W_{s}\right)_{t}$ are uniformly integrable martingales, then $\widehat{W}_{t}$ and $W_{t}^{*}$ are Brownian Motions respectively under the measures $\widehat{P}$ and $P^{*}$, defined as

$$
\frac{d \widehat{P}}{d P}=\mathcal{E}\left(-\int_{0} \lambda_{t} d W_{t}\right)_{T} \quad \text { and } \quad \frac{d P^{*}}{d P}=\mathcal{E}\left(-2 \int_{0} \lambda_{t} d W_{t}\right)_{T}
$$


We recall that $\widehat{P}$ (if it exists) is called the minimal martingale measure.

Lemma 1.15. Let $h, k$ be two predictable stochastic processes whose stochastic integrals $\int_{0}^{t} h_{s} d W_{s}^{*}$ and $\int_{0}^{t} k_{s} d M_{s}$ are defined. The following conditions are equivalent:

$$
\begin{aligned}
\exp \left(\int_{0}^{T} \lambda_{s}^{2} d s\right) & =c \frac{\mathcal{E}\left(\int_{0}^{\cdot} h_{s} d W_{s}^{*}\right)_{T}}{\mathcal{E}\left(\int_{0}^{\cdot} k_{s} d M_{s}\right)_{T}} \\
\mathcal{E}\left(-\int_{0} \lambda_{s} d W_{s}+\int_{0}^{\cdot} k_{s} d M_{s}\right)_{T} & =c \mathcal{E}\left(\int_{0}^{\cdot}\left(-\lambda_{s}+h_{s}\right) d \widehat{W}_{s}\right)_{T}
\end{aligned}
$$

where $c$ is the same constant in both equations.

Proof. We will use the properties of the Doléans exponential listed in Protter (1990) pag. 79. Starting from the left-hand side of (3), we have

$$
\begin{aligned}
\mathcal{E}\left(-\int_{0} \lambda_{s} d W_{s}+\int_{0} k_{s} d M_{s}\right)_{T} & = \\
=\mathcal{E} & \left(-\int_{0} \lambda_{s} d \widehat{W}_{s}\right)_{T} \mathcal{E}\left(\int_{0} k_{s} d M_{s}\right)_{T} \exp \left(\int_{0}^{T} \lambda_{s}^{2} d s\right)
\end{aligned}
$$

Conversely, starting from the right-hand side of (3), we have

$$
\begin{aligned}
& \mathcal{E}\left(\int_{0}\left(-\lambda_{s}+h_{s}\right) d \widehat{W}_{s}\right)_{T}= \\
&=\mathcal{E}\left(-\int_{0} \lambda_{s} d \widehat{W}_{s}\right)_{T} \mathcal{E}\left(\int_{0} h_{s} d \widehat{W}_{s}\right)_{T} \exp \left(\int_{0}^{T} \lambda_{s} h_{s} d s\right)= \\
&=\mathcal{E}\left(-\int_{0} \lambda_{s} d \widehat{W}_{s}\right)_{T} \mathcal{E}\left(\int_{0} h_{s} d W_{s}^{*}\right)_{T}
\end{aligned}
$$

The conclusion is now immediate.

From now on, we suppose that $\mathcal{M}_{e}^{2} \neq \emptyset$. By Schweizer (1996), Lemma 1 page 210, and Delbaen/Schachermayer (1996) lemma 2.2 and Theorem 1.3, we obtain the following characterization of the variance-optimal martingale measure: $\widetilde{P}$ is an element of $\mathcal{M}_{e}^{2}$ (i.e. $\widetilde{P}$ is a true probability) and it is the unique element of $\mathcal{M}_{s}^{2}$ which can be written in the form

$$
\frac{d \widetilde{P}}{d P}=c+\int_{0}^{T} \gamma_{s} d X_{s}
$$

with $c \geq 1$. In the above equation, $\gamma_{t}$ is a predictable stochastic process which does not necessarily belong to $\Theta$; however the integral process $\int_{0}^{t} \gamma_{s} d X_{s}$ is a square integrable martingale for every probability measure $Q \in \mathcal{M}_{e}^{2}$. In particular, $\int_{0}^{T} \gamma_{s} d X_{s}$ is an element of $\overline{G_{T}(\Theta)}$.

Since $\frac{d \widetilde{P}}{d P}$ is strictly positive, it can be written as a Doléans exponential. From the previous result, we obtain the following : 
Theorem 1.16. Let $h, k$ be two predictable processes such that the exponential martingale $\mathcal{E}\left(-\int_{0}^{\cdot} \lambda_{s} d W_{s}+\int_{0}^{\cdot} k_{s} d M_{s}\right)$ is square-integrable. Then $h, k$ are solutions of the equation (2) of Lemma 1.15 if and only if

$$
\frac{d \widetilde{P}}{d P}=\mathcal{E}\left(-\int_{0} \lambda_{s} d W_{s}+\int_{0}^{\cdot} k_{s} d M_{s}\right)_{T}=\frac{\mathcal{E}\left(-\int_{0}^{\cdot} \beta_{s} d X_{s}\right)_{T}}{E\left[\mathcal{E}\left(-\int_{0}^{*} \beta_{s} d X_{s}\right)_{T}\right]}
$$

where $\beta_{s}=\frac{\lambda_{s}-h_{s}}{\sigma_{s} X_{s}}$.

The equality $\frac{d \widetilde{P}}{d P}=c \mathcal{E}\left(-\int_{0} \beta_{s} d X_{s}\right)_{T}$ is useful to characterize the optimal strategy (see Rheinländer/Schweizer (1997) ); we also recall that $\beta$ is the so-called hedging numéraire of Gouriéroux et al. (1998).

\section{Explicit Solutions}

We have seen that a solution to the equation:

$$
\exp \left(\int_{0}^{T} \lambda_{s}^{2} d s\right)=c \frac{\mathcal{E}\left(\int_{0}^{\cdot} h_{s} d W_{s}^{*}\right)_{T}}{\mathcal{E}\left(\int_{0}^{\cdot} k_{s} d M_{s}\right)_{T}}
$$

provides an explicit form for the density of the variance-optimal martingale measure.

Definition 2.1. We recall the definition of the mean-variance tradeoff process $\widehat{K}_{t}$ (see, for instance, Schweizer (1996)):

$$
\widehat{K}_{t}=\int_{0}^{t} \lambda_{s}^{2} d s
$$

From (2) we can immediately see the following:

Proposition 2.2. $\widehat{K}_{T}$ is a constant if and only if $\widetilde{P}=\widehat{P}$, and $\beta=\frac{\lambda}{\sigma X}$.

This was first pointed out by Pham et al. (1998) and, for Itô processes, by Laurent/Pham (1999).

In more realistic situations, a solution to (2) can easily be found in two cases:

( $\alpha) \lambda_{s}(\omega, \eta)=\lambda_{s}(\omega)$ : in this case we set $k=0$, and solve the equation

$$
\mathcal{E}\left(\int_{0} h_{t} d W_{t}^{*}\right)_{T}=\frac{\exp \left(\int_{0}^{T} \lambda_{t}^{2} d t\right)}{E^{*}\left[\exp \left(\int_{0}^{T} \lambda_{t}^{2} d t\right)\right]}
$$

which, provided that $E^{*}$ exists, and the above expectation is finite, admits a solution by the representation property of $W$ (and thus of $W^{*}$ ) on $\Omega$. This case covers the so-called almost complete models, where $\widetilde{P}=\widehat{P}$, while $\beta_{s}=\frac{\lambda_{s}-h_{s}}{\sigma_{s}}$.

In a typical example, $H$ is an option on two observable assets, but trading is allowed in only one of them. As a result, $\mathcal{F}_{t}^{S}$ is strictly smaller than $\mathcal{F}_{t}^{W} \otimes \mathcal{E}_{t}$, unlike in the usual stochastic volatility models, where these filtrations are equal.

For a discussion on almost complete models, see for instance Pham et al. (1998) or Laurent/Pham (1999). 
We only remark that in this case $\mathcal{M}_{e}^{2} \neq \emptyset$ if and only if $\widehat{P}$ exists and $\frac{d \widehat{P}}{d P}$ is in $L^{2}$. Since $\left(\frac{d \widehat{P}}{d P}\right)^{2}=\mathcal{E}\left(-2 \int_{0} \lambda_{s} d X_{s}\right)_{T} \exp \left(\int_{0}^{T} \lambda_{t}^{2} d t\right)$, this condition is satisfied if the probability $P^{*}$ exists and $\exp \left(\int_{0}^{T} \lambda_{t}^{2} d t\right)$ is $P^{*}$-integrable.

( $\beta) \lambda_{s}(\omega, \eta)=\lambda_{s}(\eta)$ : in this case we can simply set $h=0$, and then solve the equation

$$
\mathcal{E}\left(\int_{0} k_{t} d M_{t}\right)_{T}=\frac{\exp \left(-\int_{0}^{T} \lambda_{t}^{2}(\eta) d t\right)}{E\left[\exp \left(-\int_{0}^{T} \lambda_{t}^{2}(\eta) d t\right)\right]}
$$

which always admits a solution, since $M$ has the representation property on $E$. This case covers all examples considered in Biagini/Guasoni (1999) : $\beta_{s}=\frac{\lambda_{s}}{\sigma_{s} X_{s}}$, and $\widetilde{P}$ is generally different than $\widehat{P}$, unless $\widehat{K}_{T}$ is deterministic (for diffusion processes, this is proved Pham et al. (1998), Theorem 11).

We remark that if $\int_{0}^{T} \lambda_{t}^{2}(\eta) d t$ is finite almost surely, then $\mathcal{M}_{e}^{2} \neq \emptyset$. Namely, in this case we obtain

$$
\left(\frac{d \widetilde{P}}{d P}\right)^{2}=\mathcal{E}\left(-2 \int_{0}^{\cdot} \lambda_{t}^{2}(\eta) d W_{t}\right)_{T} \frac{\exp \left(-\int_{0}^{T} \lambda_{t}^{2}(\eta) d t\right)}{E\left[\exp \left(-\int_{0}^{T} \lambda_{t}^{2}(\eta) d t\right)\right]^{2}}
$$

The process $\mathcal{E}\left(-2 \int_{0}^{\cdot} \lambda_{t}^{2} d W_{t}\right)_{T}$ is actually a stochastic integral depending on the parameter $\eta$ (see Protter (1990) for details): therefore for every fixed $\eta$ we have that $\int_{\Omega} \mathcal{E}\left(-2 \int_{0}^{\cdot} \lambda_{t}^{2}(\eta) d W_{t}\right)_{T} d P(\omega)=1$, and consequently we get

$$
E\left[\left(\frac{d \widetilde{P}}{d P}\right)^{2}\right]=\frac{1}{E\left[\exp \left(-\int_{0}^{T} \lambda_{t}^{2}(\eta) d t\right)\right]}
$$

When $\lambda_{s}(\omega, \eta)=\lambda_{s}(\eta)$, it may be hard to find $k$ explicitly ; but in fact it is often sufficient to know that it exists, since $\widetilde{P}$ can be obtained through the equality

$$
\frac{d \widetilde{P}}{d P}=\mathcal{E}\left(-\int_{0}^{\cdot} \lambda_{s} d W_{s}\right)_{T} \frac{\exp \left(-\int_{0}^{T} \lambda_{t}^{2} d t\right)}{E\left[\exp \left(-\int_{0}^{T} \lambda_{t}^{2} d t\right)\right]}
$$

Below we have some examples:

Example 2.3. If we consider the example 1.4, under the probability $\widetilde{P}$ the numbers $a_{i}$ are still independent, but $a_{i}=1$ with a new probability $\tilde{p}$, where, if $\Delta T=\frac{T}{n+1}$,

$$
\tilde{p}=\frac{p e^{-\lambda_{1}^{2}(\Delta T)}}{p e^{-\lambda_{1}^{2}(\Delta T)}+(1-p) e^{-\lambda_{2}^{2}(\Delta T)}}
$$


Example 2.4. If we consider the example 1.6, under the new probability $\widetilde{P}$ the time jump $\tau$ and the new values of $\mu$ and $\sigma$ after $\tau$ are no more independent: in Biagini/Guasoni (1999) one can find the explicit form of the law of $\tau$ under $\widetilde{P}$ and of the laws of $\mu$ and $\sigma$ conditional to $\{\tau=t\}$.

In some models, however, it may be desirable to find $k_{t}$ : this is the case, for instance, for stochastic volatility models defined by diffusion processes. In example 1.7 , if $\beta(t, x, y)=0$ and if $\alpha, \gamma, \sigma$ don't depend on $X_{t}$, we have $\lambda_{s}(\omega, \eta)=\lambda_{s}(\eta)$, and $\widetilde{P}$ can be written as in (4): however, this does not clarify the dynamics of $v_{t}$ under $\widetilde{P}$. On the other hand, if $k_{t}$ is known, then one can apply Girsanov's Theorem, and get

$$
\left\{\begin{array}{l}
d X_{t}=X_{t} \sigma\left(t, v_{t}\right) d \widetilde{W}_{t}^{1} \\
d v_{t}=\left(\alpha\left(t, v_{t}\right)-k_{t}\right) d t+\gamma\left(t, v_{t}\right) d \widetilde{W}_{t}^{2}
\end{array}\right.
$$

where $\widetilde{W}_{t}^{1}$ and $\widetilde{W}_{t}^{2}$ are independent Wiener processes under $\widetilde{P}$.

If the model is in some sense "Markovian", we obtain the following result (which coincides with Proposition 6.1 (3) of Laurent/Pham (1999), but it is proved in a completely different way) :

Proposition 2.5. Assume that $E\left[\exp \left(-\int_{t}^{T} \lambda_{s}^{2}\left(s, v_{s}\right) d s\right) \mid \mathcal{F}_{t}\right]=G\left(t, v_{t}\right)$, and that the function $G(t, x)$ is $C^{1}$ in $t$ and $C^{2}$ in $x$. Then we have

$$
\mathcal{E}\left(\int_{0} k_{s} d W_{s}^{2}\right)=\frac{\exp \left(-\int_{0}^{T} \lambda^{2}\left(s, v_{s}\right) d s\right)}{E\left[\exp \left(-\int_{0}^{T} \lambda^{2}\left(s, v_{s}\right) d s\right)\right]} \quad \text { iff } \quad k_{t}=\left.\frac{\frac{\partial G}{\partial x} \gamma}{G}\right|_{\left(t, v_{t}\right)}
$$

Proof. By martingale representation, there exists a process $g_{t}$ such that

$$
\exp \left(-\int_{0}^{T} \lambda^{2}\left(s, v_{s}\right) d s\right)=G_{0}+\int_{0}^{T} g_{s} d W_{s}^{2}
$$

Therefore:

$$
\begin{aligned}
& G_{t}=E\left[G_{T} \mid \mathcal{F}_{t}\right]=G_{0}+\int_{0}^{t} g_{s} d W_{s}^{2}= \\
&=\exp \left(-\int_{0}^{t} \lambda^{2}\left(s, v_{s}\right) d s\right) E\left[\exp \left(-\int_{t}^{T} \lambda^{2}\left(s, v_{s}\right) d s\right) \mid \mathcal{F}_{t}\right]= \\
&=\exp \left(-\int_{0}^{t} \lambda^{2}\left(s, v_{s}\right) d s\right) G\left(t, v_{t}\right)
\end{aligned}
$$

Applying Itô's formula, we obtain:

$$
d G_{t}=g_{t} d W_{t}^{2}=\exp \left(-\int_{0}^{t} \lambda^{2}\left(s, v_{s}\right) d s\right)\left(\frac{\partial G}{\partial x} \gamma\right)\left(t, v_{t}\right) d W_{t}^{2}
$$

where, in the last equality, the sum of the terms of finite variation vanishes since $G_{t}$ is a martingale. Therefore, $g_{t}=\exp \left(-\int_{0}^{t} \lambda^{2}\left(s, v_{s}\right) d s\right)\left(\frac{\partial G}{\partial x} \gamma\right)\left(t, v_{t}\right)$. However, we 
also have

$$
G_{T}=G_{0}+\int_{0}^{T} g_{s} d W_{s}^{2}=G_{0} \mathcal{E}\left(\int_{0}^{\cdot} \frac{g_{s}}{G_{s}} d W_{s}^{2}\right)_{T}=G_{0} \mathcal{E}\left(\int_{0} k_{s} d W_{s}^{2}\right)_{T}
$$

therefore $k_{t}=\frac{g_{t}}{G_{t}}$, and the proof is complete.

\section{Conditions for the Closedness of $G_{T}(\Theta)$}

The closedness of the space $G_{T}(\Theta)$ in $L^{2}(P)$ plays a key role in mean-variance hedging, since it guarantees the existence of an optimal hedging strategy in the space $\Theta$.

A sufficient condition for $G_{T}(\Theta)$ to be closed is the boundedness of $\widehat{K}_{T}$, as shown by Pham et al. (1998). In some sense, we now show that in cases $(\alpha)$ and $(\beta)$, the boundedness of $\widehat{K}_{T}$ is almost necessary. We will show that this condition is not satisfied for some commonly used models.

First we recall, and state as a theorem, a short version of a necessary and sufficient condition established by Delbaen et al. (1997) :

Theorem 3.1. Let $X$ be a continuous semimartingale: suppose that $\mathcal{M}_{e}^{2} \neq \emptyset$ and let $Z_{t}=E\left[\frac{d \widetilde{P}}{d P} \mid \mathcal{F}_{t}\right]$. The following conditions are equivalent:

i) $G_{T}(\Theta)$ is closed in $L^{2}(P)$;

ii) $Z_{t}$ satisfies the following reverse Hölder inequality:

$$
E\left[\left(\frac{Z_{T}}{Z_{\tau}}\right)^{2} \mid \mathcal{F}_{\tau}\right] \leq C
$$

for all stopping times $\tau \leq T$ and for some constant $C$.

We shall now see how this condition translates for $(\alpha)$ and $(\beta)$.

Proposition 3.2. Assume that $\mathcal{M}_{e}^{2} \neq \emptyset$ :

i) If $\lambda_{s}(\omega, \eta)=\lambda_{s}(\omega)$, then $G_{T}(\Theta)$ is closed if and only if there exists some $M$ such that, for all stopping times $\tau$,

$$
E^{*}\left[\exp \left(\int_{\tau}^{T} \lambda_{t}^{2}(\omega) d t\right) \mid \mathcal{F}_{\tau}\right]<M
$$

ii) If $\lambda_{s}(\omega, \eta)=\lambda_{s}(\eta)$, then $G_{T}(\Theta)$ is closed if and only if there exists some $\epsilon>0$ such that, for all stopping times $\tau$,

$$
E\left[\exp \left(-\int_{\tau}^{T} \lambda_{t}^{2}(\eta) d t\right) \mid \mathcal{F}_{\tau}\right]>\epsilon
$$

Proof. From 3.1, it follows that $G_{T}(\Theta)$ is closed if and only if condition ii) in Theorem 3.1 is satisfied.

For $i i$ ), we have

$$
Z_{\tau}=E\left[Z_{T} \mid \mathcal{F}_{\tau}\right]=\mathcal{E}\left(-\int_{0} \lambda_{t} d W_{t}\right)_{\tau} \frac{E\left[\exp \left(-\int_{0}^{T} \lambda_{t}^{2}(\eta) d t\right) \mid \mathcal{F}_{\tau}\right]}{E\left[\exp \left(-\int_{0}^{T} \lambda_{t}^{2}(\eta) d t\right)\right]}=
$$


It follows that

$$
\begin{gathered}
\frac{Z_{T}}{Z_{\tau}}=\frac{\mathcal{E}\left(-\int_{0}^{\cdot} \lambda_{t} d W_{t}\right)_{T}}{\mathcal{E}\left(-\int_{0} \lambda_{t} d W_{t}\right)_{\tau}} \frac{\exp \left(-\int_{0}^{T} \lambda_{t}^{2}(\eta) d t\right)}{E\left[\exp \left(-\int_{0}^{T} \lambda_{t}^{2}(\eta) d t\right) \mid \mathcal{F}_{\tau}\right]}= \\
=\mathcal{E}\left(-\int_{\tau} \lambda_{t} d W_{t}\right)_{T} \frac{\exp \left(-\int_{\tau}^{T} \lambda_{t}^{2}(\eta) d t\right)}{E\left[\exp \left(-\int_{\tau}^{T} \lambda_{t}^{2}(\eta) d t\right) \mid \mathcal{F}_{\tau}\right]}
\end{gathered}
$$

Therefore:

$$
\begin{array}{r}
E\left[\left(\frac{Z_{T}}{Z_{\tau}}\right)^{2} \mid \mathcal{F}_{\tau}\right]=\frac{E\left[\mathcal{E}\left(-2 \int_{\tau}^{\cdot} \lambda_{t} d W_{t}\right)_{T} \exp \left(-\int_{\tau}^{T} \lambda_{t}^{2}(\eta) d t\right) \mid \mathcal{F}_{\tau}\right]}{E\left[\exp \left(-\int_{\tau}^{T} \lambda_{t}^{2}(\eta) d t\right) \mid \mathcal{F}_{\tau}\right]^{2}}= \\
=\frac{E^{*}\left[\exp \left(-\int_{\tau}^{T} \lambda_{t}^{2}(\eta) d t\right) \mid \mathcal{F}_{\tau}\right]}{E\left[\exp \left(-\int_{\tau}^{T} \lambda_{t}^{2}(\eta) d t\right) \mid \mathcal{F}_{\tau}\right]^{2}}
\end{array}
$$

However, since $\lambda$ depends only on $\eta$, we find that the projection of $P^{*}$ on $\mathcal{F}_{E}$ coincides with $P$, and thus $E^{*}\left[\exp \left(-\int_{\tau}^{T} \lambda_{t}^{2}(\eta) d t\right) \mid \mathcal{F}_{\tau}\right]=E\left[\exp \left(-\int_{\tau}^{T} \lambda_{t}^{2}(\eta) d t\right) \mid \mathcal{F}_{\tau}\right]$. Hence

$$
E\left[\left(\frac{Z_{T}}{Z_{\tau}}\right)^{2} \mid \mathcal{F}_{\tau}\right]=\frac{1}{E\left[\exp \left(-\int_{\tau}^{T} \lambda_{t}^{2}(\eta) d t\right) \mid \mathcal{F}_{\tau}\right]}
$$

as claimed. For $i$ ), calculations are more straightforward:

$$
Z_{\tau}=\mathcal{E}\left(-\int_{0}^{\cdot} \lambda_{t} d W_{t}\right)_{\tau}
$$

and thus

$$
\frac{Z_{T}}{Z_{\tau}}=\frac{\mathcal{E}\left(-\int_{0}^{\cdot} \lambda_{t} d W_{t}\right)_{T}}{\mathcal{E}\left(-\int_{0}^{\cdot} \lambda_{t} d W_{t}\right)_{\tau}}=\mathcal{E}\left(-\int_{\tau}^{\cdot} \lambda_{t} d W_{t}\right)_{T}
$$

Finally

$$
\begin{aligned}
E\left[\left(\frac{Z_{T}}{Z_{\tau}}\right)^{2} \mid \mathcal{F}_{\tau}\right]=E\left[\mathcal{E}\left(-2 \int_{\tau} \lambda_{t} d W_{t}\right)_{T} \exp \left(\int_{\tau}^{T} \lambda_{t}^{2}(\omega) d t\right) \mid \mathcal{F}_{\tau}\right]= \\
=E^{*}\left[\exp \left(\int_{\tau}^{T} \lambda_{t}^{2}(\omega) d t\right) \mid \mathcal{F}_{\tau}\right]
\end{aligned}
$$

and the proof is complete.

We shall give some models where $G_{T}(\Theta)$ is not closed.

Example 3.3. Consider example 1.3 (or better the generalization of Föllmer and Leukert), where:

$$
\lambda_{t}=\lambda 1_{\left\{t<t_{0}\right\}}+\lambda(\eta) 1_{\left\{t \geq t_{0}\right\}}
$$


As mentioned before, here $E=\mathbb{R}: G_{T}(\Theta)$ is closed if and only if the distribution of $\lambda(\eta)$ has compact support.

In fact, if last condition is satisfied, then $\widehat{K}_{T}$ is bounded ; conversely, for $t \geq t_{0}$ we have

$$
E\left[\exp \left(-\int_{t}^{T} \lambda_{s}^{2}(\eta) d s\right) \mid \mathcal{F}_{t}\right]=\exp \left(-(T-t) \lambda^{2}(\eta)\right)
$$

By Proposition 3.2, the conclusion is immediate.

Example 3.4. We now examine the Heston model, that is a stochastic volatility model described by the equations

$$
\left\{\begin{array}{l}
d X_{t}=X_{t}\left(\lambda_{0} v_{t} d t+\sqrt{v_{t}} d W_{t}^{1}\right) \\
d v_{t}=\left(\alpha-\beta v_{t}\right) d t+\sqrt{v_{t}} d W_{t}^{2}
\end{array}\right.
$$

Here we have (see, for instance, Laurent/Pham (1999)) :

$$
E\left[\exp \left(-\int_{t}^{T} \lambda_{t}^{2}(\eta) d t\right) \mid \mathcal{F}_{t}\right]=\exp \left(-A(T-t) \lambda_{0}^{2} v_{t}-B(T-t)\right)
$$

where

$$
A(\tau)=\frac{1+\zeta}{\delta} \frac{1-e^{-\delta \tau}}{1+\zeta e^{-\delta \tau}} \quad \delta=\beta \sqrt{1+\frac{2 \lambda_{0}^{2}}{\beta^{2}}} \quad \zeta=\frac{\delta-\beta}{\delta+\beta}
$$

Since $\delta, \zeta>0$, it follows that $A(T-t)>0$, and therefore $(5)$ is bounded from below if and only if $v_{t}$ is bounded from above. However, this is never the case, since in Heston model $v_{t}$ is the square of a Bessel process with an appropriate change of time.

Analogous calculations can be carried out in the Stein and Stein model ( see Heath et al. (1999), example 3.2.2 for details ) showing that also in this case $G_{T}(\Theta)$ is not closed.

We point out that the drawback of the non-closedness of the space $G_{T}(\Theta)$ has been overcome by Schweizer (1999) : by exploiting the approach introduced by Gouriéroux et al. (1998), Schweizer has proved the existence of an optimal meanvariance strategy not in the space $\Theta$, but in the space $\widetilde{\Theta}$ of all predictable processes $\theta$ such that the stochastic integral $\int_{0}^{t} \theta_{s} d X_{s}$ is a square integrable martingale for every $Q \in \mathcal{M}_{e}^{2}$.

\section{Conclusions}

We have seen that a simple equation involving stochastic exponentials can identify the variance optimal probability $\widetilde{P}$ (and the mean-variance hedging strategy) in a general class of stochastic volatility models. All examples introduced are analysed in Biagini/Guasoni (1999).

We further point out that the change of numéraire approach introduced by Geman et al. (1995) can be adapted to give the explicit form of the mean-variance hedging strategy for a call option (see Biagini/Guasoni (1999)). 


\section{APPENDix}

Proposition 5.1. Any square-integrable martingale with respect to the filtration $\tilde{\mathcal{F}}_{t}$ can be written as

$$
N_{t}(\omega, \eta)=N_{0}(\eta)+\int_{0}^{t} H_{s}(\omega, \eta) d W_{s}(\omega)
$$

where $H$ is $\tilde{\mathcal{F}}_{t}$-predictable and such that $E\left[\int_{0}^{T} H_{s}^{2} d s\right]<\infty$.

Proof. Denote by $\mathfrak{M}$ the set of martingales which admit a representation in the desired form. We begin by showing that $\mathfrak{M}$ contains all martingales $N_{t}$ such that $N_{T}(\omega, \eta)=F(\omega) G(\eta)$, with $F, G$ square-integrable and measurable functions. In fact, if $F(\omega)=F_{0}+\int_{0}^{T} H_{s}(\omega) d W_{s}(\omega)$, with $E\left[F^{2}\right]=F_{0}^{2}+E\left[\int_{0}^{T} H_{s}^{2} d s\right]$, it is easily seen that:

$$
F(\omega) G(\eta)=F_{0} G(\eta)+\int_{0}^{T} H_{s}(\omega) G(\eta) d W_{s}(\omega)
$$

The stochastic process $\widetilde{H}_{s}(\omega, \eta)=H_{s}(\omega) G(\eta)$ is $\tilde{\mathcal{F}}_{t}$-predictable, and

$$
E\left[F^{2} G^{2}\right]=E\left[F_{0}^{2} G^{2}\right]+E\left[\int_{0}^{T} H_{s}^{2} G^{2} d s\right]
$$

$\mathfrak{M}$ is obvoiusly stable under linear combinations, hence the set $\left\{N_{T}: \quad N \in \mathfrak{M}\right\}$ is dense in $L^{2}\left(\Omega \times E, \mathcal{F}_{T} \otimes \mathcal{E}, P\right)$. However, if $N_{t}=N_{0}+\int_{0}^{t} H_{s} d W_{s}$, the map $N \mapsto E\left[N_{T}^{2}\right]=E\left[N_{0}^{2}+\int_{0}^{T} H_{s}^{2} d s\right]$ is an isometric injection from $\mathfrak{M}$ into $L^{2}(\Omega \times$ $\left.E, \mathcal{F}_{T} \otimes \mathcal{E}, P\right)$. This concludes the proof.

Proposition 5.2. Any square-integrable martingale $N_{t}$ with respect to the filtration $\mathcal{F}_{t}^{W} \otimes \mathcal{E}_{t}=\mathcal{F}_{t}$ can be written in the form

$$
N_{t}(\omega, \eta)=N_{0}+\int_{0}^{t} H_{s}(\omega, \eta) d W_{s}(\omega)+\int_{0}^{t} K_{s}(\omega, \eta) d M_{s}(\eta)
$$

with $H, K$ predictable and such that:

$$
E\left[\int_{0}^{T} H_{s}^{2} d s+\int_{0}^{T} K_{s}^{2} d[M]_{s}\right]<\infty
$$

Proof. Denote by $\mathfrak{M}$ the set of martingales which admit a representation in the desired form. We start showing that $\mathfrak{M}$ contains all martingales $N_{t}$ such that $N_{T}(\omega, \eta)=F(\omega) G(\eta)$. We write $F(\omega)=F_{0}+\int_{0}^{T} H_{s}(\omega) d W_{s}(\omega), G(\eta)=G_{0}+$ $\int_{0}^{T} K_{s}(\eta) d M_{s}(\eta)$, and consider the martingales $R_{t}=E\left[F \mid \mathcal{F}_{t}\right]$ and $V_{t}=E\left[G \mid \mathcal{F}_{t}\right]$. By Itô's formula, and recalling that $[W, M]=0$, we have

$$
F(\omega) G(\eta)=F_{0} G_{0}+\int_{0}^{T} V_{s} H_{s} d W_{s}+\int_{0}^{T} R_{s} K_{s} d M_{s}
$$

Again, $\mathfrak{M}$ is stable under linear combinations, and $\left\{N_{T}: N \in \mathfrak{M}\right\}$ is dense in $L^{2}$. Since the map $N \mapsto E\left[N_{0}^{2}+\int_{0}^{T} H_{s}^{2} d s+\int_{0}^{T} K_{s}^{2} d[M]_{s}\right]$ is an isometric injection from $\mathfrak{M}$ in $L^{2}$, the proof is complete. 


\section{REFERENCES}

[1] Biagini F. and Guasoni P. (1999) "Mean-variance hedging with random volatility jumps", Preprint.

[2] Delbaen F., Monat P., Schachermayer W., Schweizer M. and Stricker C. (1997) "Weighted norm inequalities and hedging in incomplete markets" Finance and Stochastics $\mathbf{1}$ (3) , 181-227

[3] Delbaen F. and Schachermayer W. (1996) "The variance-optimal martingale measure for continuous processes" Bernoulli 2, 81-105

[4] Dellacherie C. and Meyer P.A. (1982) "Probabilities and Potential B: Theory of martingales" North-Holland, Amsterdam.

[5] Duffie D. and Richardson H. L. (1991) "Mean-variance hedging in continuous time" Annals of Applied Probability 1, 1-15

[6] Föllmer H. and Leukert P. (1999) "Quantile hedging" Finance and Stochastics 3(3) , 251-274

[7] Föllmer H. and Schweizer M. (1991) "Hedging of contingent claims under incomplete information" In R.J. Elliot and M.H.A. Davis, editors, Applied Stochastic Analysis 389-414. Gordon and Breach.

[8] Föllmer H. and Sondermann D. (1986) "Hedging of non-redundant contingent claims" In W. Hildebrand and A. Mas-Colell, editors, Contribution to Mathematical Economics, 205-223.

[9] Geman H., El Karoui N. and Rochet J.C. (1995) "Changes of numéraire, changes of probability measures and option pricing" J. Appl. Probab. 32, 443-458

[10] Gouriéroux L., Laurent J.P. and Pham H. (1998) "Mean-variance hedging and numéraire" Math. Finance 8 , 179-200.

[11] Harrison J.M. and Pliska R.S. (1981) "Martingales and Stochastic Integrals in the Theory of continuous trading" Stoc. Processes and their Appl. 11, 215-260.

[12] Heath D., Platen E. and Schweizer M. (1999) "A comparison of Two Quadratic Approaches to hedging in Incomplete Markets" Preprint

[13] Laurent J.P. and Pham H. (1999) "Dynamic programming and mean-variance hedging" Finance and Stochastics 3(1), 83-110.

[14] Pham H., Rheinländer T. and Schweizer M. (1998) "Mean-variance hedging for continuous processes: new proofs and examples" Finance and Stochastics 2(2) , 173-198.

[15] Protter P. (1990) "Stochastic Integration and Differential Equations: A new approach" Springer-Verlag.

[16] Rheinländer T. and Schweizer M. (1997) "On $l^{2}$-projections on a space of stochastic integrals" Annals of Probability 25 (4) , 1810-1831

[17] Schäl M. (1994) "On quadratic cost criteria for option hedging" Mathematics of Operations Research $19,121-131$.

[18] Schweizer M. (1992) "Mean-variance hedging for general claims" Annals of Applied Probability 2, 171-179.

[19] Schweizer M. (1996) "Approximation pricing and the variance-optimal martingale measure" Annals of Probability 64, 206-236.

[20] Schweizer M. (1999) "A Guided Tour through Quadratic Hedging Approaches" Preprint.

[21] Zangari P. (1996) "An improved metodology for measuring VaR" Risk-Metrics Monitor 2 , $7-25$.

Dip. di Matematica Piazza Porta S. Donato 40100 Bologna Italy

E-mail address: biagini@dm.unibo.it

Scuola Normale Superiore P.zZa dei Cavalieri, 756126 Pisa Italy

E-mail address: guasoni@sns.it

Dip. di Matematica Via Buonarroti, 256127 Pisa Italy

E-mail address: pratelli@dm.unipi.it 